\title{
Análisis Crítico de la Literatura. Un Proceso Elemental en el Ejercicio de la Práctica Clínica Basada en la Evidencia
}

\author{
Critical Analysis of Literature. A Basic Process in Exercising Evidence Based Clinical Practice
}

\author{
Claudia Asenjo-Lobos* \& Carlos Manterola******
}

\begin{abstract}
ASENJO-LOBOS, C. \& MANTEROLA, C. Análisis Crítico de la Literatura. un proceso elemental en el ejercicio de la práctica clínica basada en la evidencia. Int. J. Morphol., 32(3):950-955, 2014.

RESUMEN: Para realizar una práctica clínica basada en la evidencia (PCBE), se requiere adquirir una serie de habilidades; entre ellas la búsqueda de literatura en forma sistemática y eficiente y la aplicación de reglas formales para la evaluación de la literatura encontrada. De este modo, se logra obtener de forma precisa la mejor evidencia disponible. Es así como, se podría resumir que el proceso de PCBE incluye varios pasos, uno de los cuales es el análisis crítico de la evidencia encontrada en la búsqueda de la literatura. Es fundamental comprender que no toda la información proveniente de revistas científicas es confiable o verdadera y por ende adecuada para ser instaurada como guía para la práctica clínica. Por tal razón, la información debe ser analizada de forma crítica; es decir, examinar cuidadosa y sistemáticamente la investigación para juzgar su validez y confiabilidad; o dicho de otra forma, su valor y relevancia en un contexto particular. El objetivo de este artículo fue jerarquizar la información existente respecto de estrategias para realizar un análisis crítico de la literatura científica y describir las herramientas más utilizadas con este fin.
\end{abstract}

PALABRAS CLAVE: Análisis crítico de la literatura; Lectura crítica; Guías de usuario; Validez interna; Validez externa.

\section{INTRODUCCIÓN}

Hace 20 años, nace la práctica clínica basada en la evidencia (PCBE), con el objetivo de dar solución a problemas clínicos (Guyatt et al., 2008) y David Sackett, uno de los creadores de este paradigma, la define en 1992, como el uso racional, explícito, juicioso y actualizado de la mejor evidencia para la toma de decisiones en el cuidado de un paciente (Evidence-Based Medicine Working Group, 1992).

La PCBE considera que la intuición, la experiencia clínica no sistemática, y la justificación fisiopatológica no constituyen motivo suficiente para la toma de decisiones clínicas; haciendo hincapié en el estudio de la evidencia existente proveniente de la investigación clínica (Guyatt et al., 2000). Esto no quiere decir que se trate solo de basarse únicamente en las investigaciones, se trata de integrar la mejor evidencia disponible, con la experiencia clínica y las preferencias del paciente (Sackett et al., 2000).

Para una PCBE, se requiere adquirir una serie de habilidades; entre ellas la búsqueda de literatura en forma sistemática y eficiente y la aplicación de reglas formales para la evaluación de la literatura encontrada (Guyatt et al., 2000).
De este modo, se logra obtener de forma precisa la mejor evidencia disponible.

Es así como, se podría resumir que el proceso de PCBE incluye cinco pasos: Formulación de un pregunta contestable para resolver un problema clínico, búsqueda de la mejor evidencia disponible aplicable al problema; análisis crítico de la evidencia existente en relación a validez, relevancia clínica y aplicabilidad (Hill \& Spittlehouse, 2001); interpretación y aplicación de los resultados de la evidencia en el entorno clínico correspondiente; y evaluación de la prestación realizada (Dawes et al., 2005) (Fig. 1).

El objetivo de este artículo es jerarquizar la información existente respecto de estrategias para realizar un análisis crítico de la literatura científica y describir las herramientas más utilizadas con este fin.

Definición de Análisis Crítico de la Literatura. Es fundamental comprender que no toda la información que proviene de revistas científicas puede llegar a ser confiable o verdadera y consecuentemente adecuada para ser instaurada 


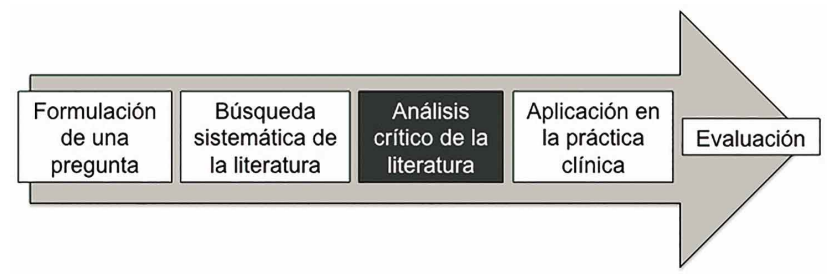

Fig. 1. Los pasos esenciales en proceso de la PCBE.

como guía para la practica clínica (Abdullah \& Firmansyah, 2012). Por tal razón, la información debe ser analizada críticamente; es decir, examinar cuidadosa y sistemáticamente la investigación para juzgar su validez y confiabilidad; o dicho de otra forma, su valor y relevancia en un contexto particular (Burls, 2009).

Con ese fin, fue que en 1981, en el Departamento de Epidemiología Clínica y Bioestadística de la Universidad de McMaster, se elaboraron las primeras guías de lectura; las que bajo el apelativo de "Readers' Guides", comenzaron a ser utilizadas para analizar críticamente los artículos referentes a diagnóstico, pronóstico, etiología y tratamiento.

Posteriormente en un número de la revista JAMA del año 1992, se publicaron las modificaciones a las guías de lectura; las que se basaron en la necesidad de dar un enfoque más práctico al análisis de la literatura biomédica. Es decir, que éstas se orientaran a resolver los problemas de la práctica clínica cotidiana, en definitiva de los pacientes. Se trabajó entonces desde la perspectiva de la PCBE, lo que involucra la habilidad de acceder a la validez e importancia de la evidencia antes de aplicarla a los problemas clínicos del día a día. Esto, fue lo que determinó que el concepto de "Readers' Guides" se sustituyese por el de "Users' Guides" (Evidence-Based Medicine Working Group).

Existen varias pautas que permiten analizar críticamente en forma ordenada y sistemática los artículos primarios referentes a tratamiento y prevención, etiología y daño (causalidad o riesgo), pronóstico e historia natural, y diagnóstico; y para valorar artículos integradores, secundarios y de sinopsis como son las revisiones sistemáticas de la literatura (RS), los estudios económicos, los análisis de decisiones, las guías de práctica clínica y los estudios de calidad de vida relacionada a la salud (CVRS) (Manterola, 2009).

Las primeras preguntas que debiéramos hacernos para descartar algunos artículos son relacionadas a la pregunta que esta siendo revisada o estudiada. En este punto cobran interés dos aspectos, la pregunta y el diseño de investigación. definida?

¿Existe una pregunta de investigación claramente
Lo esperable es que el (los) autor (es) mencione (n) la pregunta de investigación que esta (n) investigando y quiere (n) responder. Esta puede deducirse tanto del objetivo como de la hipótesis. En el reporte puede ser encontrada en el resumen o en el último párrafo de la introducción (Greenhalgh, 2010).

¿Cuál es el diseño de investigación usado?

Hay diseños ideales, adecuados y menos adecuados para dar respuesta a cada pregunta y a cada escenario de estudio. Por otra parte, el diseño del estudio empleado puede influir en la fuerza de la recomendación y es generalmente de naturaleza jerárquica (Fig. 2).

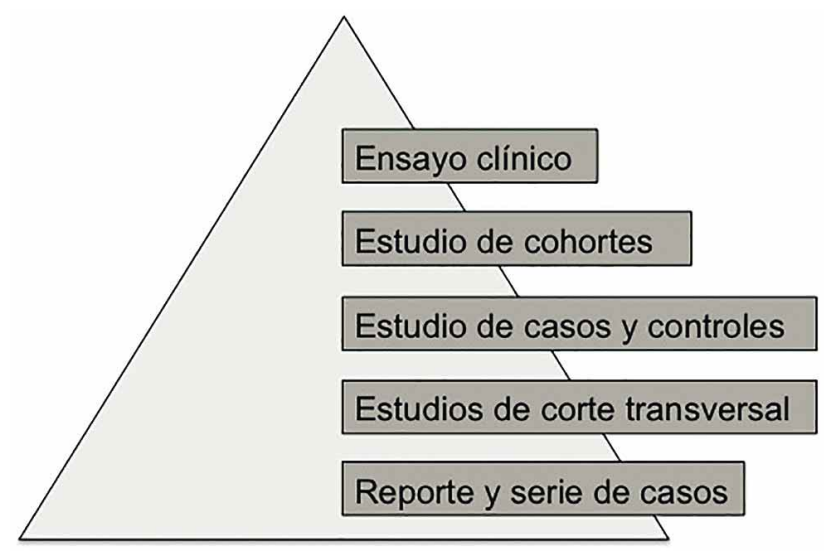

Fig. 2. Los diseños adecuados a cada escenario y pregunta de investigación.

¿Fue el diseño de investigación apropiado para responder la pregunta a la pregunta?

Es muy frecuente que una pregunta pueda ser contestada por más de un diseño de investigación, por lo que cabe preguntarse y analizar si el diseño utilizado es el mejor para dirigir la investigación; y si no, comprender cuales son las otras opciones (Greenhalgh, 2010; Young \& Solomon, 2009).

En la Tabla I se observan los tipos de preguntas que es posible encontrar según escenario clínico y además el tipo de diseño de investigación ideal que permite responder estas preguntas. Si alguna de estas 3 preguntas no es respondida o su respuesta es no, no vale la pena someter el articulo al análisis de su validez utilizando alguna guía de análisis critico. En caso contrario, si nos convencemos de que el artículo vale la pena leerlo, pasamos a la etapa de la evaluación crítica de este. Para ello, se han de evaluar tres aspectos fundamentales: la validez interna, el impacto y la validez externa. Las guías de análisis crítico disponibles se basan en estos tres criterios. 
Tabla I. Tipos de preguntas según escenario y diseño del estudio.

\begin{tabular}{|c|c|c|}
\hline Escenarios & Preguntas & $\begin{array}{l}\text { Tipo de diseños ideales para } \\
\text { conducir el estudio }\end{array}$ \\
\hline Causalidad o Riesgo & $\begin{array}{l}\text { ¿Se asocia la exposición a uno o varios } \\
\text { factores con un aumento en el riesgo de } \\
\text { presentar un desenlace indeseado? }\end{array}$ & $\begin{array}{l}\text { RS de estudios de cohorte, estudios de } \\
\text { cohorte individuales, EC con asignación } \\
\text { aleatoria. }\end{array}$ \\
\hline Diagn óstico & $\begin{array}{l}\text { ¿El empleo de una prueba diagnóstica } \\
\text { conlleva un aumento en la probabilidad } \\
\text { de detectar o de descartar una condición } \\
\text { clínica? }\end{array}$ & $\begin{array}{l}\text { RS de estudios de pruebas diagnósticas, } \\
\text { estudio de pruebas diagnósticas } \\
\text { individuales, estudios de casos y controles. }\end{array}$ \\
\hline Terapia & $\begin{array}{l}\text { ¿El uso de una intervención profiláctica } \\
\text { o terapéutica implica una reducción en } \\
\text { el riesgo de padecer una enfermedad o } \\
\text { evento de interés en sujetos libres de } \\
\text { ella; o de presentar resultados } \\
\text { indeseables en quienes ya la padecen? }\end{array}$ & $\begin{array}{l}\text { RS de EC con asignación aleatoria, EC con } \\
\text { asignación aleatoria in dividuales, estudios } \\
\text { de cohortes. }\end{array}$ \\
\hline Pronóstico & $\begin{array}{l}\text { ¿Cuáles son las consecuencias (buenas } \\
\text { y malas) esperadas en individuos que } \\
\text { tienen una condición clínica } \\
\text { determinada? }\end{array}$ & $\begin{array}{l}\text { RS de estudios de cohorte, estudios de } \\
\text { cohorte individ uales, EC con asignación } \\
\text { aleatoria. }\end{array}$ \\
\hline
\end{tabular}

Validez interna. Se define como el grado en el que los datos obtenidos al medir un fenómeno representan la verdadera dimensión de lo medido (Fletcher \& Wagner, 2005). Se refiere a la medida en que los métodos y análisis de estudio permiten llegar a conclusiones apropiadas acerca del grupo estudio evaluado (Skelly, 2012). Es decir, que cuanto más válido es un estudio, sus resultados se aproximan más a la verdad (Manterola). La validez interna está presente cuando las posibles explicaciones alternativas (por ejemplo, el azar, la confusión y el sesgo) de los resultados del estudio han sido minimizadas o excluidas por lo que es posible obtener conclusiones adecuadas.

Una medición precisa, no sesgada y análisis adecuados son esenciales para la validez interna. Todos los estudios (incluso los EC) tienen un potencial de sesgo. La clave es determinar la medida en que los potenciales sesgos po- drían influir en la solidez de los resultados y la capacidad de extraer conclusiones significativas (Cardozo-Montilla, 2011). Las diferencias sistemáticas entre los grupos de estudio es el principal factor que pone en riesgo a la validez interna de un estudio, lo que conlleva a reportar resultados erróneos (Skelly).

Sesgo es un error sistemático que puede conducir a una estimación inexacta de la verdadera asociación entre la exposición y la enfermedad o evento de interés (EI). En la Tabla II, se resumen los principales tipos de sesgos que es posible encontrar en los estudios publicados y que deberían evitarse durante la ejecución de un estudio (Hennekens \& Buring, 1987; De la Guarda et al. 2012). Por otra parte, en la Tabla III se enumeran los procedimientos que indican que se han tomado medidas para controlar potenciales sesgos que son reportados en las investigaciones.

Tabla II. Tipos de sesgos que se pueden encontrar en los estudios.

\begin{tabular}{|c|c|c|}
\hline Tipo de Sesgo & Definición & Causa \\
\hline Sesgo de selección & $\begin{array}{l}\text { Diferencias entre aquellos que participan en el } \\
\text { estudio y quienes no (población blanco). } \\
\text { La muestra no es representativa. }\end{array}$ & 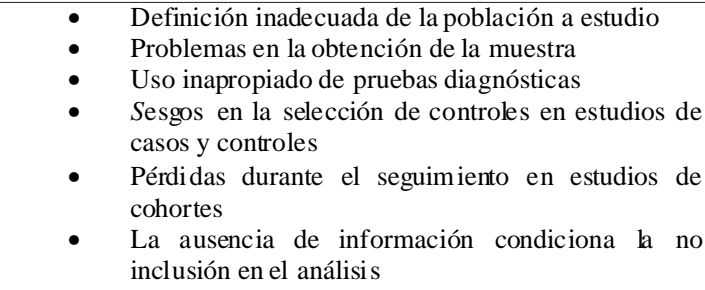 \\
\hline Sesgo de Información & $\begin{array}{l}\text { Diferencias de la calidad de la información entre } \\
\text { grupos de comparación debido a fallas en el } \\
\text { método de recolección de datos. }\end{array}$ & $\begin{array}{l}\text { Sesgo del entre vistador u observador: Cuando el entrevistador } \\
\text { dirige las preguntas buscando confirmar la hipótesis } \\
\text { Sesgo del recuerdo: Cuando los sujetos en estudio exageran la } \\
\text { información o no las recuerda en forma correcta }\end{array}$ \\
\hline Sesgo de Confusión & $\begin{array}{l}\text { Existencia de una tercera variable que puede } \\
\text { causar la enfermedad o evento de interés y que a } \\
\text { la vez se asocia al Factor de riesgo en estudio. }\end{array}$ & $\begin{array}{l}\text { Una variable confundente debe tener } 3 \text { características: } \\
\text { - Ser factor de riesgo para la enfermedad o evento de } \\
\text { interés. } \\
\text { - } \quad \text { Estar asociada a la exposición. } \\
\text { No ser un paso intermedio entre factor de riesgo y } \\
\text { enfermedad o evento de interés. }\end{array}$ \\
\hline
\end{tabular}


Otro error, es el error aleatorio definido como el grado de incertidumbre de los resultados, que no se puede evitar ya que es imposible predecir y cuantificar su impacto. La mejor manera de reducir el error aleatorio es mediante la estimación correcta del tamaño de la muestra que se requiere para comprobar la hipótesis (De la Guarda et al.).

Impacto clínico del resultado. El impacto clínico de los resultados depende del tamaño y la precisión del efecto reportado en el artículo. El tamaño del efecto se trata de valorar la magnitud del este; la que se mide a través de la diferencia de medias o de medianas (cuando se trata de variables continuas); el cálculo del riesgo relativo y la reducción absoluta de riesgo, (cuando se trata de variables dicotómicas); la razón de probabilidades, el número necesario a tratar, y el número necesario a dañar (Herbert, 2000a y 2000b).

Por otra parte, la precisión del efecto, consiste evaluar la variabilidad de los datos que dan origen a la medida de efecto. Para ello, es preciso determinar los intervalos de confianza del 95\%, que corresponde al rango de valores (calculado en una muestra), en el cual se encuentra el verdadero valor del parámetro, con una probabilidad determinada; probabilidad que se denomina nivel de confianza. Es así como un intervalo muy amplio, nos indica que existe mucha variabilidad en los datos y los resultados son poco precisos (Sim \& Reid, 1999).

Validez externa. La validez externa o aplicabilidad, se refiere a la utilidad del artículo analizado en nuestra práctica clínica; o a la reproducibilidad de los resultados en el medio en que se desenvuelve el lector. Es decir, el grado de similitud de los pacientes incluidos, de la intervención realizada, la consideración de todos los resultados clínicamente relevantes en el estudio, el análisis de los beneficios respecto a perjuicios y costos de la intervención en estudio (incluidos las preferencias del paciente) (Manterola).

Hay que evaluar y analizar si existen diferencias so- ciales y económicas, fisiológicas o epidemiológicas de nuestros pacientes en relación a los participantes del estudio que estamos evaluando. Como regla general, se debe pensar si nuestros pacientes hubiesen estado en el lugar donde se realizó el estudio hubiesen sido seleccionados a participar según criterios de inclusión y exclusión del estudio; y si es así, es posible que los resultados sean aplicables (Dans et al., 1998). No olvidar que si un estudio no posee validez interna, la validez externa no relevante (Skelly).

De este modo, los aspectos antes señalados se pueden resumir en tres preguntas presentes en todas las guías: ¿Son válidos los resultados del estudio? ¿Cuáles son los resultados? ¿Podrán los resultados ayudar en el cuidado de mis pacientes?

Éstas, aplican para artículos referentes a tratamiento y prevención; artículos de relativos a diagnóstico y etiología, morbilidad o complicaciones, y pronóstico e historia natural. Existen los denominados "artículos integradores"; que no son otra cosa que estudios en los que se trabaja con la evidencia generada a partir de estudios primarios (como los mencionados en el párrafo anterior), la que es en definitiva analizada, integrada y finalmente resumida. En este ámbito, se dispone también de guías de usuario para RS, análisis de decisiones, guías de práctica clínica, evaluaciones económicas y estudios de CVRS.

Las guías y herramientas para realizar análisis crítico de la literatura para los distintos escenarios de estudio están disponibles en las direcciones que se resumen en la Tabla IV.

El análisis crítico de la literatura es el factor más importante para poder llevar la evidencia a la práctica en forma precisa. Es necesario desarrollar nuevas habilidades como la identificación de la pregunta de los autores, conocer los diseños de investigación, identificar posibles sesgos, interpretar los resultados, y analizar la aplicabilidad de los hallazgos al cuidado de los pacientes en particular.

Tabla III. Procedimientos que indican que se han tomado medidas para controlar potenciales sesgos.

\begin{tabular}{|c|c|}
\hline Tipo de Sesgo & Control de sesgo \\
\hline Sesgo de selección & $\begin{array}{ll}\text { - } & \text { Muestra aleatorizada. } \\
\text { - } & \text { Pérdidas en el seguimiento menor del } 20 \% \text { de la muestra. }\end{array}$ \\
\hline Sesgo de información & $\begin{array}{l}\text { - Fuentes y m étodos de información deben ser semejante para los grupos } \\
\text { estudio. } \\
\text { Entrevistadores y personal de estudio enmascarados al tratamiento o } \\
\text { exposición. } \\
\text { - Pérdidas en el seguimiento menor del } 20 \% \text { de la muestra. }\end{array}$ \\
\hline Sesgo de confusión & $\begin{array}{l}\text { - } \quad \text { Control de confundentes a traves de análisis multivariados. } \\
\text { - } \quad \text { Análisis estratificados. } \\
\text { - Emparejamiento en estudios de casos-controles. }\end{array}$ \\
\hline
\end{tabular}


Tabla IV. Guías y Herramientas para Realizar Análisis Crítico de la Literatura para los Distintos Escenarios y sus Respectivas Direcciones Electrónicas.

\begin{tabular}{|c|c|c|c|}
\hline Fuente & Localización & Herramientas & Categoría de estudio \\
\hline $\begin{array}{l}\text { CASP } \\
\text { (Critical } \\
\text { Appraisal Skills } \\
\text { Programme) }\end{array}$ & $\begin{array}{l}\text { http://www.casp-uk.net/find- } \\
\text { appraise-act/appraising-the- } \\
\text { evidence }\end{array}$ & $\begin{array}{l}\text { Se encuentran las diferentes guías para } \\
\text { análisis critico en inglés. }\end{array}$ & $\begin{array}{l}\text { EC, RS, estudios de cohortes, estudios de casos y } \\
\text { controles. } \\
\text { Investigación cualitativa. } \\
\text { Evaluaciones económicas. } \\
\text { Estudios de pruebas diagnósticas. } \\
\text { Reglas de predicción. }\end{array}$ \\
\hline CASPe & $\frac{\mathrm{http}: / / \text { www.redcaspe.org/drupa }}{1 / ? \mathrm{q}=\text { node/32 }}$ & $\begin{array}{l}\text { Guías de análisis crítico en español. } \\
\text { Calculadoras. } \\
\text { Glosario. }\end{array}$ & Lo mi smo que el anterior. \\
\hline CEBM & http://www.cebm.net $/ ? o=1040$ & $\begin{array}{l}\text { Guías de análisis crítico en español, } \\
\text { inglés y alemán. } \\
\text { Calculadoras. } \\
\text { CATmaker. }\end{array}$ & $\begin{array}{l}\text { EC, RS, estudios de pruebas diagnósticas, } \\
\text { estudios de pronóstico }\end{array}$ \\
\hline JAMA evidence & $\frac{\text { http://www.hopkinsmedicine.o }}{\text { rg/gim/training/Osler/osler_JA }}$ & Guías análisis crítico en inglés. & $\begin{array}{l}\text { Terapia o prevención, pruebas diagnósticas, } \\
\text { d año, pronóstico, análisis de decisión, } \\
\text { investigac ión cualitativa, guías clínicas, calidad } \\
\text { de vida, estudios económi cos. }\end{array}$ \\
\hline
\end{tabular}

ASENJO-LOBOS, C. \& MANTEROLA, C. Critical analysis of literature. A basic process in exercising evidence based clinical practice. Int. J. Morphol., 32(3):950-955, 2014.

SUMMARY: To carry out clinical practice based on evidence (CPBE), it is necessary to acquire some skills, including systematic literature search and application of formal rules for evaluation of the literature found, thereby obtaining the best evidence available. Thus, one could summarize the CPBE process includes several steps, one of which is critical appraisal of the evidence found in literature search. It is essential to understand that not all the information from scientific journals is reliable or valid, and therefore suitable to be instituted in clinical practice. For this reason, the information must be critically analyzed; i.e. carefully and systematically examining research to judge its validity and reliability, or stated another way, its value and relevance in a particular context. The aim of this article is to hierarchy the existing information on strategies to realize critical analysis of the scientific literature and to describe the tools most commonly used for this purpose.

KEY WORDS: Critical appraisal of biomedical articles; Evidence-Based Practice; Reader's Guides; Users' Guides.

\section{REFERENCIAS BIBLIOGRÁFICAS}

Abdullah, M. \& Firmansyah, M. Critical appraisal on journal of clinical trials. Acta Med. Indones. 44(4):337-43, 2012

Burls, A. What is critical appraisal? Hayward Medical Communications. 2009. Disponible en: http:// www.medicine.ox.ac.uk/bandolier/painres/download/whatis/ what_is_critical_appraisal.pdf.

Cardozo-Montilla, M. A. Evaluación crítica de la literatura biomédica. Validez y aplicabilidad de la información científica en el contexto de la práctica clínica basada en evidencia. Cienc. Odontol., 8(1):57-67, 2011.
Dans, A. L.; Dans, L. F.; Guyatt, G. H. \& Richardson, S. Users' guides to the medical literature: XIV. How to decide on the applicability of clinical trial results to your patient. EvidenceBased Medicine Working Group. JAMA, 279(7):545-9, 1998.

Dawes, M.; Summerskill, W.; Glasziou, P.; Cartabellotta, A.; Martin, J.; Hopayian, K.; Porzsolt, F.; Burls, A.; Osborne, J. $\&$ Second International Conference of Evidence-Based Health Care Teachers and Developers. Sicily statement on evidence-based practice. BMC Med. Educ., 5(1):1, 2005.

De la Guarda, G.; Gómez, M. L.; Sandoval, E. \& García, G. Sesgo o error de medición. En: Villa, A. Epidemiología y Estadística en Salud Pública. México, McGraw-Hill, 2012. pp.151-63. 
Evidence-Based Medicine Working Group. Evidence-based medicine. A new approach to teaching the practice of medicine. JAMA, 268(17):2420-5, 1992.

Fletcher, R. H. \& Wagner, E. H. Diagnosis. In: Fletcher, R. H.; Fletcher, S. W. \& Wagner, E. H. Clinical epidemiology. 4th ed. Baltimore, Ed. Lippincott Williams \& Wilkins, 2005.

Greenhalgh, T. How to read a paper: the basics of evidence-based evidence. 4th ed. London, Wiley-Blackwell, 2010.

Guyatt, G. H; Naylor, D.; Richardson, W. S.; Green, L.; Haynes, R. B.; Wilson, M. C.; Cook, D. J. \& Jaeschke, R. Z. What is the best evidence for making clinical decisions? JAMA, 284(24):3127-8, 2000.

Guyatt, G.; Rennie, D.; Meade M. O. \& Cook, D. J. Users' Guides to the Medical Literature: A Manual for Evidence-Based Clinical Practice. 2nd ed. American Medical Association \& McGraw-Hill Education, 2008. pp. 809. Disponible en: http:/ /jamaevidence.com/resource/520.

Hennekens, C. H. \& Buring, J. E. Epidemiology in medicine. Boston, Little Brown and Company, 1987.

Herbert, R. D. Critical appaisal of clinical trials. I: estimating the magnitude of treatment effects when outcomes aremeasured on a continuous scale. Aust. J. Physiother, 46:229-235, 2000.

Herbert, R. D. Critical appraisal of clinical trials. II. estimating the magnitude of treatment effects when outcomes are measured on a dichotomous sacle. Aust. J. Physiother., 46:309-13, 2000.

Hill, A. \& Spittlehouse, C. What is critical appraisal? Hayward Medical Communications. 2001. Disponible en: http:// meds.queensu.ca/medicine/obgyn/pdf/what_is/ WhatisCriticalAppraisal.pdf.

Manterola, C. Analisis crítico de la literatura biomédica. Rev. Med. Clin. Las Condes, 20:371-81, 2009.

Sackett, D. L; Straus, S. E.; Richardson, W. S.; Rosenberg, W. \& Haynes, R. B. Evidence-Based Medicine: How to Practice and Teach EBM. 2nd ed. Edinburgh, Churchill Livingstone, 2000.

Sim, J. \& Reid, N. Statistical inference by confidence intervals: issues of interpretation and utilization. Phys. Ther., 79:18695, 1999.

Skelly, A. C. Class of evidence (CoE): how does it relate to critical appraisal? Evid. Based Spine Care J., 3(2):7-10, 2012.

Young, J. M \& Solomon, M. J. How to critically appraise an article. Nat. Clin. Pract. Gastroenterol. Hepatol., 6(2):82-91, 2009.

\author{
Dirección para Correspondencia: \\ Prof. Dr. Carlos Manterola \\ Departamento de Cirugía y Traumatología \\ Universidad de La Frontera \\ Manuel Montt 112, Oficina 408 \\ Temuco \\ CHILE
}

Email: carlos.manterola@ufrontera.cl

Recibido: 22-05-2014

Aceptado: 18-06-2014 